\title{
Application of Regenerative Braking with Optimized Speed Profiles for Sustainable Train Operation
}

\author{
Leon Allen ${ }^{1}$ and Steven Chien (iD) ${ }^{1,2}$ \\ ${ }^{1}$ Department of Civil and Environmental Engineering, New Jersey Institute of Technology, NJ 07102, Newark, USA \\ ${ }^{2}$ School of Transportation Engineering, Chang'an University, Xi'an 710064, China \\ Correspondence should be addressed to Steven Chien; chien@njit.edu
}

Received 28 April 2021; Revised 23 June 2021; Accepted 16 August 2021; Published 6 September 2021

Academic Editor: Keping Li

Copyright (c) 2021 Leon Allen and Steven Chien. This is an open access article distributed under the Creative Commons Attribution License, which permits unrestricted use, distribution, and reproduction in any medium, provided the original work is properly cited.

\begin{abstract}
This paper presents a method for synergizing the energy-saving strategies of integrated coasting and regenerative braking in urban rail transit operations. Coasting saves energy by maintaining motion with propulsion disabled, but it induces longer travel time. Regenerative braking captures and reuses the braking energy of the train and could shorten travel time but reduces the time available for coasting, indicating a tradeoff between the two strategies. A simulation model was developed based on fundamental kinematic equations for assessing sustainable train operation with Wayside Energy-Saving Systems (WESSs). The objective of this study is to optimize speed profiles that minimize energy consumption, considering the train schedule and specifications, track alignment, speed limit, and the WESS parameters such as storage limit and energy losses in the transmission lines. The decision variables are the acceleration at each time step of the respective motion regimes. Since the study optimization problem is combinatorial, a Genetic Algorithm was developed to search for the solution. A case study was conducted which examined various scenarios with and without WESS on a segment of an urban rail transit line to test the applicability of the proposed model and to provide a platform for the application of ideas developed in this study. It was determined that synergizing the energy-saving strategies of coasting and regenerative braking yielded the greatest efficiency of the scenarios examined.
\end{abstract}

\section{Introduction}

Electric rail vehicles have been known to have high energy efficiencies compared to the other modes of transportation and play a leading role in sustainability. This is in part due to their large hauling capacities and the low rolling resistance between the steel-to-steel wheel/rail boundary [1]. However, they consume an inordinate amount of energy due to the scale of operations and frequency of service, even though they are the least energy-intensive mode [2].

Transportation accounts for $28 \%$ of energy consumption [3] and 28.2\% of Greenhouse Gas Emissions [4] in the USA. Of this amount, road vehicles are responsible for $83.6 \%$ of the total carbon emissions by the transportation sector as against $2.3 \%$ by rail transportation vehicles [5]. This suggests that rail vehicles have one of the lowest carbon dioxide $\left(\mathrm{CO}_{2}\right)$ emission levels among major transportation modes.
However, by improving the efficiency of the operation, energy consumption and $\mathrm{CO}_{2}$ emissions could be further reduced thereby reducing the costs to the operator and protecting the environment. Modern railcars are either diesel or electric powered. Although electric trains do not emit pollutants locally, there was $60 \%$ of electricity generated derived from fossil fuels $[6,7]$. Therefore, electric trains indirectly incur significant carbon emissions which can be reduced by reducing energy consumption.

The objective of this study was to develop a model to optimize train speed profile, which minimizes energy consumption with the integration of the coasting and regenerative braking energy-saving strategies. A Wayside EnergySaving System (WESS) was installed to supply peak power to the trains on acceleration and to store regenerated energy on braking. In this way, the operator avoids drawing peak power from the utility and the associated elevated energy 
charges and provides a receptacle for the regenerated energy, lest it is dissipated as heat via the braking resistors.

The main contribution of this study is the optimization of the speed profiles of the train under various scenarios with and without WESS, which minimize energy consumption with coasting and regenerative braking, considering various factors including train schedule and specifications (e.g., train weight, passenger weight, and motor power), track alignment, and the WESS parameters (i.e., storage capacity and line losses). The decision variables were the acceleration rates at the time steps in various motion regimes (i.e., acceleration, cruising, coasting, and braking) considering maximum allowable speed and scheduled travel time. Since the study optimization problem is combinatorial, a Genetic Algorithm was developed to search for the solution. A simulation model was developed based on fundamental kinematic equations for assessing sustainable train operation with Wayside Energy-Saving Systems (WESS).

\section{Review of Literature}

More than $60 \%$ of total rail energy consumed is absorbed by traction $[8,9]$ including a considerable amount consumed by the traction motors on initial acceleration [10]. Therefore, energy efficiency could be improved by aggressively targeting areas with a high potential for reduction. GonzalezGil et al. [11] developed a set of Key Performance Indicators (KPIs) to monitor different aspects of energy consumption. Brandstrom [12] achieved a 5\% saving by redistributing the run times of the train between stations through drive style optimization and schedule improvement.

Generally, operators focus on either programmed driving or energy recovery for reducing consumption and mitigating environmental degradation. Energy recovery could involve either storage in an energy storage receptacle or timetable optimization where train arrivals and departures are coordinated for efficient use of regenerative braking energy. In programmed driving, the driver is informed of the most energy-efficient throttle positions using the optimized speed profiles. Kim and Chien [13] developed an optimization model in which they adjusted the length of the coasting regime considering the maximum train speed and reduced the energy consumption by $30 \%$ overruns where there was no energy-saving strategy, albeit the resulting travel time increased by 7\%. Yang et al. [14] achieved increases in line capacity, 25\% energy savings, and 5 to $15 \%$ increase in traffic capacity. Allen and Chien [15] developed a model to reduce energy consumption with optimal coasting while maintaining the schedule for a multistation rail segment. Liu et al. [16] proposed a method to maximize the use of recovered braking energy through timetable optimization. With the use of an Improved Artificial Bee Colony (IABC) algorithm, they saved $40.1 \%$ energy over an unoptimized timetable.

A popular method, the maximum principle, involves optimal control theory, where the traction, braking, and resistance forces which satisfy the velocity, acceleration, travel distance, and trip time constraints are determined. Howlett [17] explored the options of discrete and continuous control to determine the switching points of the coasting and braking regimes. Liu and Golovitcher [18] approximated the energy consumed by calculating the motion regimeswitching points of the speed trajectory. They developed a program for achieving the sequence of controls for optimizing train operation and schedules. Other studies adopted unorthodox approaches for the optimization of energy consumption. Su et al. [19] considered the use of excess dwell time in the previous travel segment of the train to supplement the next segment by leaving earlier and arriving on schedule. This operation saved energy by lowering the average speed. Zhu et al. [20] proposed an energy distribution method which adopted a Q-learning approach to optimize the driving strategies. Energy units were added to different sections of the speed profile to reduce trip time but adhere to the constraints. Their method could be applied to both stochastic and deterministic environments. However, these studies did not consider regenerative braking as an energysaving strategy which could result in significant savings on the energy consumed by rail vehicles.

Some studies have gravitated toward combinatorial solutions with the aim of achieving benefits from two or more strategies and used various solution algorithms to achieve optimization. Gong et al. [21] synchronized dwell time to allow an accelerating train to use regenerative energy from a train braking within the same electrical section while combining timetable optimization with the energy-efficient operation to minimize energy consumption. Su et al. [22] integrated driving strategy and timetable optimization to reduce energy drawn from the substation and increase regenerative braking energy use, while Wang et al. [23] achieved almost $50 \%$ consumption improvement by using a dynamic programming (DP) model to obtain a global solution and a discrete differential dynamic programming (DDDP) algorithm to reduce computation time. Liu et al. [24] proposed a nonlinear programming model to maximize the utilization of regenerated energy harvested from braking trains by accelerating trains using dwell time, headway, and length of service time as constraints. The energy that was not absorbed by an accelerating train was stored in a WESS. They also used a hybrid algorithm with fixed dwell time to verify their model and achieved almost an $82 \%$ utilization ratio of regenerated energy. Luan et al. [25] presented a study where they integrated a train dispatching method with a train control method to optimize the train speed profiles. Huang et al. [26] designed a dynamic programming model to jointly optimize the running time and speed profiles of a metro line with on-board energy storage for the braking energy. Dominguez et al. [27] designed a model to optimize the speed profiles to reduce the traction energy in a system with automatic train operation (ATO) using different energy storage options.

None of the studies discussed above explicitly utilized coasting as an energy-saving strategy, considering the losses occurring in the transmission line. Coasting saves energy by maintaining motion with traction disabled so that it does not require additional energy consumption. Transmission line losses may seem minor for each interstation traveled, but are additive, and could be significant in the long term. Allen and 
Chien [28] achieved energy savings of more than 20\% through combined coasting and regenerative braking strategies using a mathematical method. However, for this type of multidimensional problem, Genetic Algorithms were found to be efficient in finding a solution.

\section{Methodology}

3.1. Model Formulation. The aim of the proposed dynamic model is to compute the energy consumption of a train varying with the speed along the track over time. The acceleration was assumed to be constant within each time step (0.01 seconds). Consequently, kinematic equations were developed for the acceleration, cruising, and braking regimes. The model uses Newton's laws of motion to compute the speed, distance, acceleration, energy, and travel time values. These are then input into a GA to synergize the strategies of coasting and regenerative braking according to methods developed by the authors.

3.2. Acceleration. Acceleration of the train occurs when there is an imbalance in the horizontal forces acting on it. The forces typically acting on an accelerating train are the train resistance $R^{t}$, the braking force $B^{t}$, the equivalent mass $M_{e}$, the normal reaction to its weight $N^{t}$, and tractive effort $F^{t}$. The train cannot be in a powering and braking mode at the same time, and thus, the tractive effort and braking force cannot be simultaneously applied.

The acceleration $a^{t}$ of a train at time $t$ is formulated in equation (1) as the tractive effort $F^{t}$ less the resistance $R^{t}$ and then divided by the equivalent mass of the train $M_{e}$. Thus,

$$
a^{t}=\frac{F^{t}(v)-R^{t}(v)}{M_{e}}, \quad \forall t .
$$

The speed and cumulative distance traveled in the next time step are represented by equations (2) and (3), respectively:

$$
\begin{aligned}
& v^{t+1}=v^{t}+a^{t} t, \quad \forall t, \\
& s^{t+1}=s^{t}+\left(\frac{v^{t}+v^{t+1}}{2}\right) t, \quad \forall t .
\end{aligned}
$$

The unit train resistance $R_{u}^{t}$ is represented by equation (4) suggested by the Association of American Railroads [2]:

$$
R_{u}^{t}=\left(0.65+10 G^{t}+\frac{129}{w}+0.009 v^{t}+0.0716 A\left(v^{t}\right)^{2}\right), \quad \forall t,
$$

where $G^{t}$ is the percentage gradient at time $t, w$ is the weight per axle (tons), $v^{t}$ is the speed at time $t$, and $A$ is the crosssectional area of the train. Therefore, the total train resistance is the product of the unit resistance, the weight per axle, and the number of axles denoted as $n$. Thus,

$$
R^{t}=R_{u}^{t} \cdot w \cdot n, \quad \forall t .
$$

The applied tractive effort $F^{t}$ between the wheels and the running rails at time $t$ is the minimum of the force exerted by the traction motors, denoted as $F_{m}^{t}$, and the adhesive force, denoted as $F_{a}^{t}$, formulated as equations (6) and (7), respectively.

$$
F_{m}^{t}=\frac{375 \eta P_{r}}{v^{t}}, \quad \forall t
$$

375 is a conversion factor, $\eta$ is the motor efficiency, $P_{r}$ is the motor power, and $v^{t}$ is the train speed at time $t$ [29].

$$
F_{a}^{t}=\mu^{t} M_{e} \cos \theta^{t}, \quad \forall t,
$$

where $\mu^{t}$ is the coefficient of adhesive friction and $\theta^{t}$ is the inclination angle of the train to the horizontal at time $t$. Therefore, the mechanical power consumed at time $t$ can be expressed by

$$
P^{t}=\frac{v^{t} \cdot F^{t}}{375 \eta}, \quad \forall t
$$

The incremental energy consumed by the train at time $t$ is given by

$$
e^{t}=P^{t} \frac{t}{3600} \cdot \frac{1}{1.341}, \quad \forall t,
$$

where the 3600 converts seconds to hours, and the coefficient of 1.341 converts horsepower to kilowatts. Finally, the energy consumed during acceleration denoted as $E_{a}$ is formulated as

$$
E_{a}=\sum_{t=0}^{t_{a}} e^{t}, \quad \forall t,
$$

where $t_{a}$ is the duration for the acceleration regime.

3.3. Cruising. In the cruising regime, the speed remains constant subject to the maximum operating speed. The tractive forces effectively balance the resistive forces. Thus,

$$
F^{t}=R^{t}, \quad \forall t .
$$

The energy consumed is the sum of the respective amounts consumed during $t_{c}$. Thus,

$$
E_{c}=\sum_{t=t_{a}}^{t_{c}} e^{t}, \quad \forall t .
$$

The total energy consumed without the WESS denoted as $E_{T}^{\prime}$ is equivalent to that consumed during the acceleration and cruising regimes. Therefore,

$$
E_{T}^{\prime}=E_{a}+E_{c} .
$$

3.4. Braking. The braking rate $b^{t}$ is equal to the sum of the braking force $B^{t}$ and train resistance divided by the equivalent mass of the train $M_{e}$. Thus,

$$
b^{t}=\frac{B^{t}+R^{t}}{M_{e}}, \quad \forall t,
$$

where $B^{t}$ is equal in magnitude to the adhesive force stated in equation (7). On application of the brakes, the motors are 
forced to run as generators thereby converting the kinetic energy of the train to electrical energy. Equation (15) is a representation of the energy regenerated at time $t$ when the train brakes [30]:

$$
e_{r}^{t}=\left[0.01072 m \rho\left[\left(v^{t}\right)^{2}-\left(v^{t+1}\right)^{2}\right]+27.25\left(s^{t+1}-s^{t}\right) m G^{t}-\left(0.2778 R^{t}\left(s^{t+1}-s^{t}\right)\right)\right] \eta_{r}, \quad \forall t,
$$

where $e_{r}^{t}$ is the regenerated energy, $G^{t}$ is the percentage gradient experienced by the train at time $t, m$ is the mass of the train in tons, $R^{t}$ is the train resistance, $s^{t}$ is the incremental distance traveled, and $\eta_{r}$ is the regeneration coefficient. This coefficient, which is taken to be 0.82 in this study, compensates for the inefficiencies of the charge and discharge cycles of the WESS.

3.5. Transmission Line Losses. During consumption and regenerative braking, energy is lost due to dissipation in the electrical resistance of the transmission line segments. Considering the location of a train between a pair of stations with spacing $S$ at time $t$, the distance to the downstream stop is $s^{t}$ and thus the distance to the upstream stop is $\left(S-s^{t}\right)$. Since a WESS is positioned at each of the stations, the electrical resistance between the train and each WESS conforms with the following piecewise equation:

$$
r^{t}=\left\{\begin{array}{ll}
s^{t} r_{u}, & \text { for } s^{t} \leq 0.5 \mathrm{~S}, \\
\left(S-s^{t}\right) r_{u}, & \text { for } s^{t}>0.5 \mathrm{~S},
\end{array} \quad \forall t\right.
$$

where $r^{t}$ is the line resistance at time $t$ and $r_{u}$ is the resistance per foot of the transmission line.

The power lost at time $t$ denoted as $P_{L}^{t}$ is the product of the square of the current $\left(I^{t}\right)$ drawn and line resistance $r^{t}$, where $I^{t}$ is equal to the power consumed $P^{t}$ divided by the line voltage $V$. Thus,

$$
P_{L}^{t}=\left(\frac{P^{t}}{V}\right)^{2} \cdot r^{t}, \quad \forall t
$$

Finally, the energy dissipated in the transmission line denoted as $e_{L}^{t}$ at time $t$ is

$$
e_{L}^{t}=P_{L}^{t} . t, \quad \forall t
$$

The total energy returned to the network through regenerative braking is defined as the total energy regenerated less the total transmission line losses. Thus,

$$
E_{r}=\sum_{t=t_{c}}^{t_{b}} e_{r}^{t}-\sum_{t=0}^{t_{b}} e_{L}^{t}, \quad \forall t .
$$

The objective is to minimize net consumed energy, denoted as $E_{T}$, which is equal to the sum of total energy consumed by train operation $E_{T}^{\prime}$ and energy returned from WESS $E_{r}$. Thus,

$$
E_{T}=\min \left(E_{T}^{\prime}-E_{r}\right), \quad \forall t .
$$

The decision variable of $E_{T}$ is the acceleration rate at each time step, which was optimized subject to maximum allowable speed, station spacing, and scheduled travel time expressed by equations (21)-(23), respectively.

$$
\begin{aligned}
v^{t} & \leq v_{\max }, \quad \forall t, \\
\sum_{t=0}^{T} s^{t} & =S, \quad \forall t \\
\left(t_{a}+t_{c}+t_{c o}+t_{b}\right) & \leq T, \quad \forall t .
\end{aligned}
$$

\section{Solution Algorithm}

The problem at hand was a complex undertaking involving time-varying input variables. Due to its combinatorial nature, a Genetic Algorithm (GA) was applied to search for the optimal solution. GA starts with a randomly generated initial population formed with a priori knowledge of the problem. In this way, the optimization starts with a set of initial solutions for faster convergence; then, the following genetic operators are applied sequentially: Reproduction (Selection), Crossover, and Mutation. The fitness value is to be minimized and, in this case, is equal to $E_{T}$ in equation (20).

4.1. Genetic Algorithm. The process begins with a randomly generated initial population consisting of a set of individuals, each of which is a potential solution to the problem. Each individual consists of a set of cells or genes describing each motion regime. Each cell contains a vector describing a characteristic of the train in each time step, which in this case is 0.01 seconds. For instance, the acceleration for the entire interstation movement can be described as

$$
a=\left[\begin{array}{llllll}
a_{1} & a_{2} & a_{3} & \ldots & a_{l-1} & a_{l}
\end{array}\right],
$$

where $l$ is the total number of time steps and each $a_{i}(i \in 1,2,3 \ldots l)$ is the acceleration rate at the $i^{\text {th }}$ time step. The distance traveled during the acceleration regime could be similarly described as

$$
s=\left[\begin{array}{llllll}
s_{1} & s_{2} & s_{3} & \ldots & s_{l-1} & s_{l}
\end{array}\right],
$$

and the duration of the acceleration regime as the scheduled travel time $T$.

A series of cells in the chromosome contain the acceleration rates in a series of time steps of various speed regimes (i.e., acceleration, cruising, coasting, and braking). The length of the chromosome denoted as $l$, also the number of cells, is equal to scheduled travel time $T$ divided by the duration of time step $\Delta T$. Thus, 


$$
l=\frac{T}{\Delta T}
$$

In cases where the train arrives at its destination ahead of schedule, the chromosome will be of the same length as stated in equation (26), but the cells for the time steps that exceed the actual travel time would be empty.

4.1.1. Reproduction. This involves the selection of strings with above average properties from the current population and inserting them into the mating pool based on the probability of them producing even better offspring. A string is selected with a probability that is proportional to its fitness.
4.1.2. Crossover. The crossover frequency is used to discover a promising region for convergence. A low crossover rate slows convergence, while a high crossover rate leads to saturation around one solution. The crossover operation randomly selects two individuals from the mating pool and exchanges portions of the strings, creating new strings. In the following example, for two parent strings (Parent 1 and Parent 2), if the crossover is on the third digit, they would exchange the digits after the third and yield Offspring 1 and Offspring 2.

$$
\begin{aligned}
& \text { (Parent 1) } X_{1}=\{010010 \mid 1001101\} \\
& \left(\text { Parent 2) } X_{2}=\{101101 \mid 0010010\}\right.
\end{aligned} \Rightarrow \begin{aligned}
& \text { (Offspring 1) } X_{3}=\{010010 \mid 0010010\} \\
& \left(\text { Offspring 2) } X_{4}=\{101101 \mid 1001101\}\right.
\end{aligned}
$$

4.1.3. Mutation. In this operation, the diversity of the strings is ensured, and premature convergence is prevented. A child string is produced from a single parent string by inverting a digit in a randomly selected position, that is, changing a one to zero or vice versa. As shown hereinafter, the fifth digit is the mutation point in the old string and is shown as the boldfaced " 0 ." This digit is shown inverted as a boldfaced " 1 " in the new string.

$$
\text { Old string }\{110001001100\} \text {, }
$$

New string $\{110011001100\}$.

Note that a high mutation rate may lead to instability, while a low rate may introduce difficulty in reaching a solution and cause the process to be trapped in local optima.

4.1.4. Population Size. When formulating a GA, the population size is of extreme importance. Smaller populations could result in less accurate solutions but less computation time. Increasing the population size could mean simultaneous handling of many solutions and could increase computation time but may increase the accuracy of the result. However, this would increase the likelihood of convergence to a global optimum because of the increased number of available solutions.

In this study, the mutation rate is set to 0.01 , probability of mutation 0.1 , and probability of crossover 0.8 . The GA options are the user-selected termination criteria, for which a population size of 10 was used, in addition to a maximum generation number of 20, 8 for maximum stall generations, and a maximum run time of 1000 seconds.

The steps taken to optimize the energy consumed are listed as follows and conform with the flowchart in Figure 1.

(1) Input train specifications and alignment parameters into the simulator at time $t=0$; set the maximum allowable trip time $T$ and station spacing $S$
(2) Calculate the train resistance $R^{t}$ using equation (5), tractive effort $F^{t}$ using equations (6) and (7), and the minimum braking distance $b_{c r}$ by inserting the brake rate $\left(b^{t}\right)$ from equations (14) into (3); then accelerate the train to maximum allowable speed

(3) Check if current travel speed has reached the maximum allowed and conduct a cruising regime if needed; if not, return to Step 2

(4) Check if elapsed time and distance are greater than those allotted for the cruising regime and conduct a coasting regime if needed; otherwise, return to Step 3

(5) Check if the speed reaches the coasting termination speed and determine the required braking rate; otherwise, return to Step 4

(6) Check the travel distance $R_{D}$ remaining to arrive at the next station. Conduct a braking regime and regenerate the braking energy to the WESS if $R_{D}$ is less than the minimum braking distance $b_{c r}$; otherwise, return to Step 5

(7) Terminate the simulation and report the speed profiles if the train arrived at the next station; otherwise, return to Step 6

(8) Output the optimal speed profile, coasting termination speed $V_{c}$, the energy consumed $E_{T}$, the energy regenerated $E_{r}$, and total travel time $T$.

\section{Case Study}

There are 40 westbound daily trains serving stations Jamaica and East New York, approximately 5 miles apart, on Long Island Rail Road's Hempstead branch. This segment is chosen to assess the performance of the proposed model and the benefits of WESS. The number of passengers varies at different times of the day, and this has a great impact on energy consumption. Of the 40 trains, there are 14 peakhour trains with an average ridership of 1500 passengers/ 


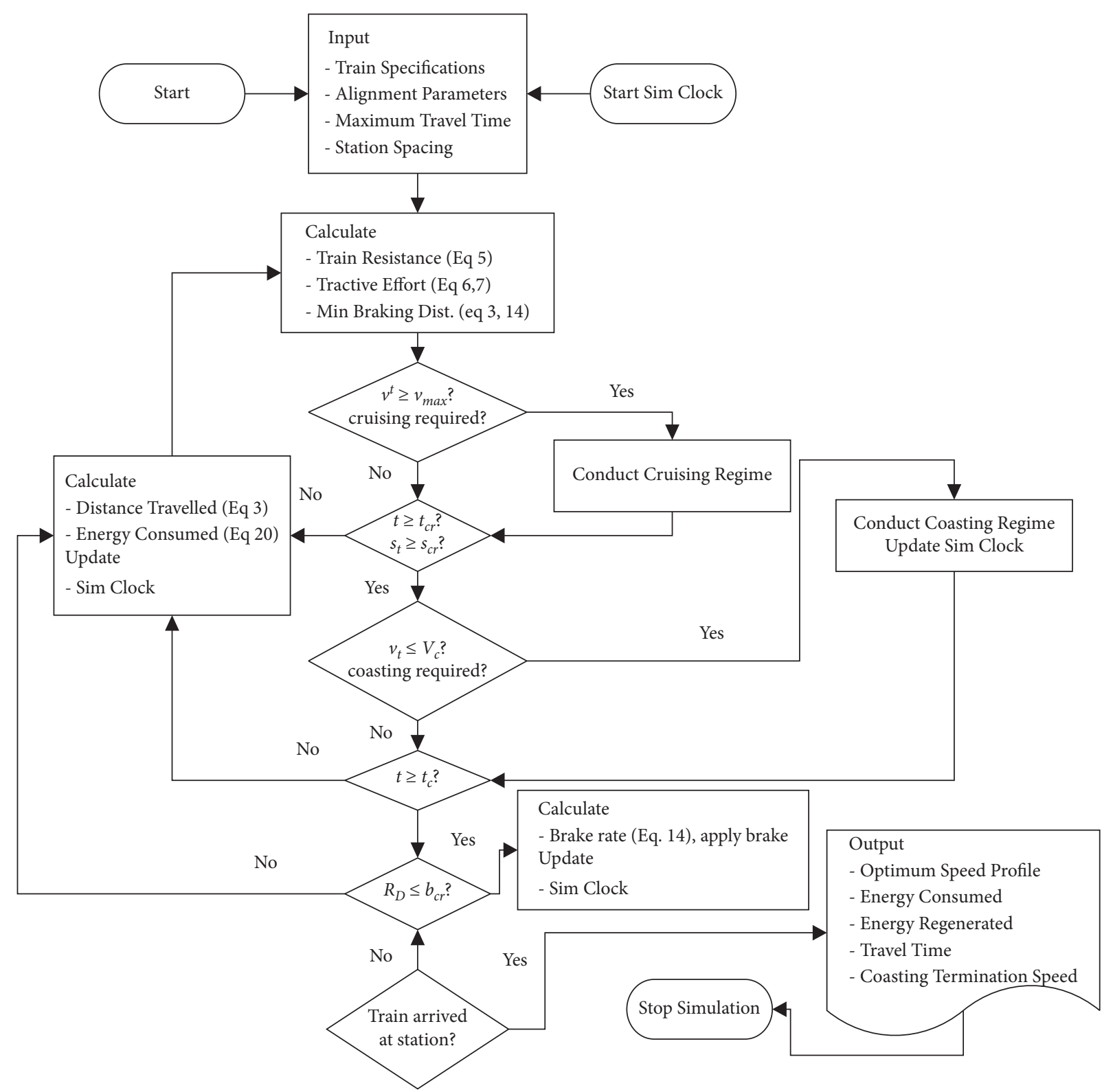

Figure 1: Flowchart for simulation of the train movement.

train and 26 off-peak trains with 750 passengers/train. Peak periods occur between 6:00 am and 10:00 am and between 4: $00 \mathrm{pm}$ and 8:00 pm on weekdays, while off-peak periods are all other times on weekdays, weekends, and holidays. The benefits of WESS were assessed on a weekday basis. The alignment topography over the study segment is represented in Figure 2, consisting of two stations at approximately equal elevations separated by a convex parabolic spacing. The train specifications are listed in Table 1.

\section{Results and Discussion}

In this section, the simulation analysis was conducted under various scenarios for peak operation:

(i) Scenario I (Baseline run): Travel time was minimized without applying coasting and regenerating braking. The train was accelerated to the maximum operating speed where it remained until the brakes had to be applied to stop at the next station.

(ii) Scenario II (coasting only): The train was accelerated to the maximum operating speed; then, it maximized the coating regime before the brakes had to be applied for the train to stop at the next station.

(iii) Scenario III (regenerative braking only): The train was operated like Scenario I. However, on the application of the brakes, the regenerated energy was captured and stored in a WESS for later reuse.

(iv) Scenario IV (coasting and regenerative braking): Regenerative braking was combined with coasting in a synergistic manner to optimize the energy consumed by the train. 


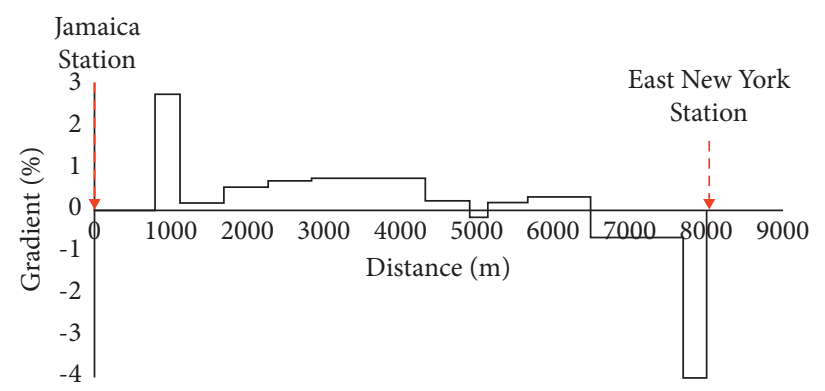

Figure 2: Alignment gradient vs. distance.

TABle 1: Train specifications.

\begin{tabular}{|c|c|c|c|}
\hline Parameters & Values & Parameters & Values \\
\hline Number of cars per train & $10 \mathrm{cars} /$ train & Maximum operating speed & $120 \mathrm{~km} / \mathrm{h}$ \\
\hline Number of traction motors & 4 units/car & Approximate headway & $2640 \mathrm{~s}$ \\
\hline Motor power & $265 \mathrm{hp}$ & Maximum acceleration rate & $3.2 \mathrm{~km} / \mathrm{h} / \mathrm{s}$ \\
\hline Weight per car & $63,045 \mathrm{~kg}$ & Maximum deceleration rate & $4.8 \mathrm{~km} / \mathrm{h} / \mathrm{s}$ \\
\hline \multirow{3}{*}{$\begin{array}{l}\text { Passenger weight } \\
\text { Coefficient of rotating masses }\end{array}$} & $122,727 \mathrm{~kg}$ & Air resistance coefficient & 0.07 \\
\hline & $61,364 \mathrm{~kg}$ & Regeneration coefficient & 0.82 \\
\hline & 1.04 & & \\
\hline
\end{tabular}

The peak-hour optimal speed profiles are shown in Figure 3(a), where travel speed is plotted against distance and indicates to the train driver the speeds at which the train could be safely operated, given the remaining trip distance. Scenario II with the lowest average speed incurs the longest travel time, followed by Scenario IV.

The plots for Scenarios I and III are superimposed on each other since they both operate according to the shortest travel time, which translates to the fastest average speed and is shown in Figure 3(b). The only difference between them is that Scenario III includes the regenerative braking feature, which does not affect the travel time but incurs less energy consumption.

The comparative cumulative energy consumption for the four scenarios is illustrated by the energy profiles in Figure 3(c), where the energy consumption is plotted against the distance traveled.

For Scenarios I and II where no regenerative braking is applied, the sections of the plot for the acceleration and cruising regimes are represented by positively sloping sections followed by horizontal sections representing the cruising (if included) and braking regimes. In Scenarios III and IV, the acceleration and cruising regimes are also represented by upward sloping sections on the plots and a horizontal one for cruising. The regenerative braking energy, being energy returned to the system, is regarded as a negative consumption and is represented by the negatively sloping sections of the plots after the cruising regimes.

According to Table 2, for the peak operation, Scenario IV consumed $44 \%$ of the energy consumed in Scenario I, but the incurred travel time was 13\% longer. Scenarios II and III consumed $45 \%$ and $67 \%$ of the energy consumed in Scenario I, respectively, and the travel time for Scenario II was 18\% greater than that of Scenario I. Since the inclusion of regenerative braking does not affect travel time, the travel times for Scenarios I and III were identical. In addition, since, in railroad operations, punctuality is a priority, Scenarios II and IV with the highest travel times would only be included when the train is running ahead of schedule, even though they consume the least energy.

In the off-peak operation, speed profiles yielded were similar to those obtained with the peak period passenger volume since the added passenger weight is minimal compared with the train weight. Scenario IV consumed $43 \%$ of the energy consumed in Scenario I, but its travel time was $20 \%$ greater. Scenario IV and Scenario II consumed similar amounts of energy and incurred similar travel times. The energy consumed by Scenario IV was 57\% less than that consumed by Scenario III, but its travel time was $20 \%$ greater.

Generally, without the WESS (Scenarios I and II), travel time plays an important role in energy consumed by the train. For instance, with Scenario II, as travel time for a particular travel segment is increased, more coasting is added to the operation which results in reduced consumption. On the other hand, the inclusion of the WESS brought a significant reduction in energy consumption by synergizing coasting and regenerative braking, thereby reducing the cost to the operator and increasing the sustainability of the service. The train undergoes a shorter coasting regime and utilizes regenerative braking to optimize the operation. For instance, WESS installation (Scenario IV) resulted in less energy consumption at the off-peak and peak periods, respectively, than when coasting was the only option (Scenario II) to reduce energy consumption.

In summary, the inclusion of WESS would reduce travel time and save energy especially during the peak-hour operation, which allows for increased capacity and elevates the level of service (LOS). 


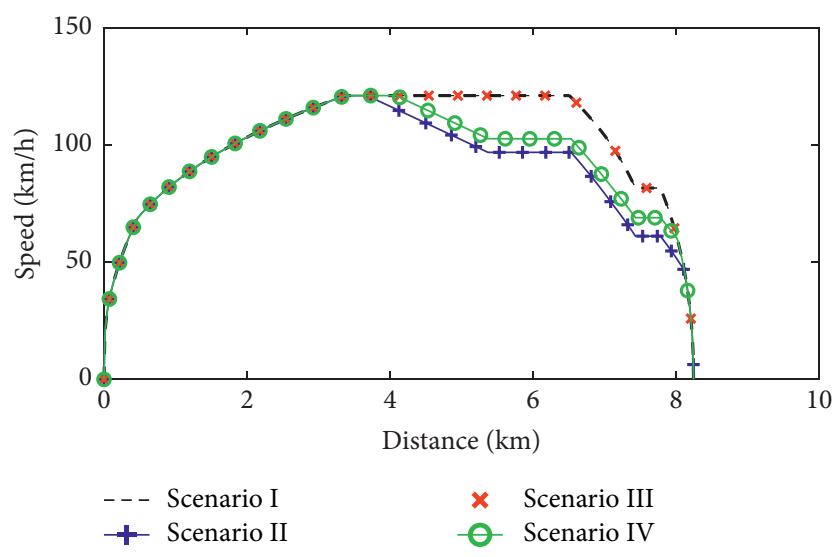

(a)

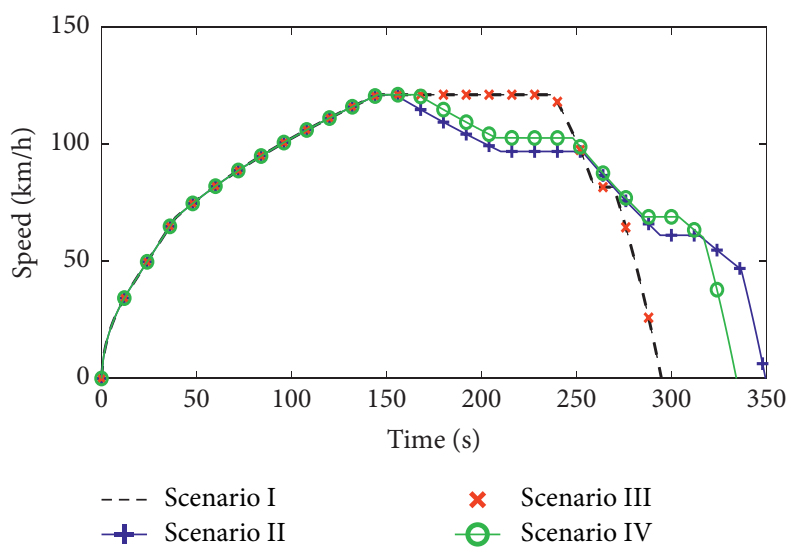

(b)

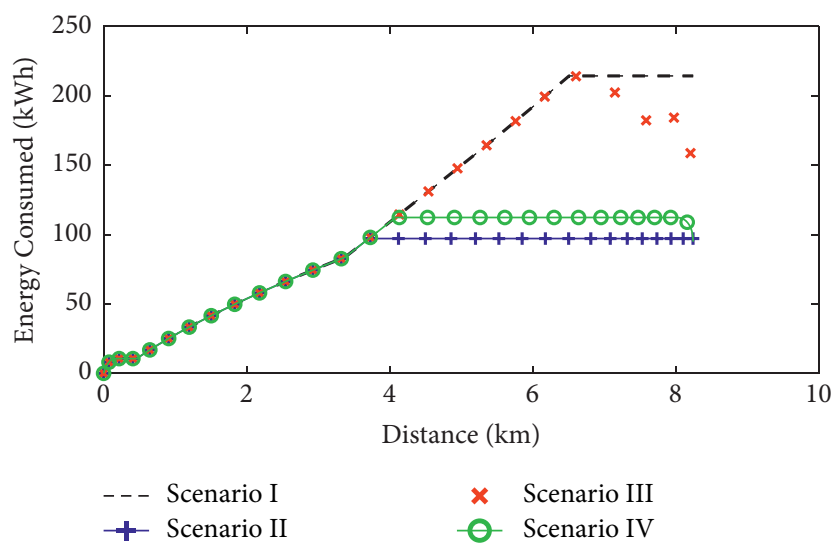

(c)

Figure 3: Optimal speed and energy profiles over space and time for various scenarios (peak). (a) Optimal speed profiles. (b) Speed/time plots. (c) Optimal energy profiles.

TABLE 2: Optimized results with various scenarios during peak and off-peak periods.

\begin{tabular}{lcccc}
\hline Scenario & Peak & & \multicolumn{2}{c}{ Off-peak } \\
& Energy consumed $(\mathrm{kWh})$ & Travel time $(\mathrm{s})$ & Energy consumed $(\mathrm{kWh})$ & Travel time $(\mathrm{s})$ \\
\hline I & 214.4 & 294.8 & 207.7 & 290.8 \\
II & 97.2 & 349.5 & 99.5 & 349.1 \\
III & 144.1 & 294.8 & 143.1 & 290.8 \\
IV & 95.1 & 334.2 & 91.1 & 349.8 \\
\hline
\end{tabular}

Note. Train unladen weight 753,182 kg; peak passenger volume 1500 passengers; off-peak passenger volume 750 passengers.

6.1. Sensitivity Analysis. The following section examines the input and output of the model and the processes occurring in between and identifies the key parameters in the input that would influence the value of the output. In this instance, changes in the optimized speed profiles (the outputs) are observed when maximum speeds, passenger volumes at peak and off-peak periods, and maximum allowable travel times were varied.

6.1.1. Energy Consumption and Travel Times vs. Maximum Speed (Peak Periods). Figure 4 displays the optimum speed profiles obtained from the simulation for various maximum operating speeds. These plots indicate, to the train driver, the different maximum speeds that can be reached and the different speeds that should be maintained for the train to safely travel while minimizing energy consumption. These optimal speed profiles could be used for trains to recover from service delays that are typical with adverse weather conditions, track maintenance, or signal malfunctions.

It is normal for electric traction motors to exert higher tractive efforts at lower speeds, resulting in higher energy consumption. The tractive effort decreases exponentially with increases in the operating speed. Therefore, as the maximum operating speed increases, the overall energy consumed decreases since, in addition to exerting less tractive effort, the train can apply coasting where possible to save energy. On the other hand, at lower operating 


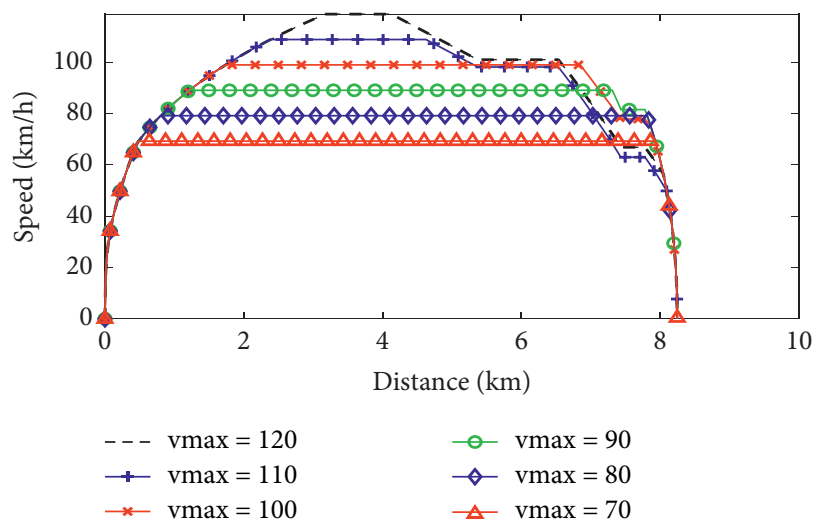

FIgURE 4: Optimized speed profiles for various maximum speeds (peak).

speeds, the increased energy consumption against tractive effort is sustained for a longer period in the cruising regime so that the train does not violate the maximum allowable travel time constraint. This is also because, at lower speeds, there is little or no opportunity to apply coasting as could be seen in Figure 4 in instances where $v_{\max }$ values are 70 and $80 \mathrm{~km} / \mathrm{h}$. In addition to reduced tractive effort causing reduced energy consumption at increased maximum operating speeds, the opportunities for prolonged coasting if the train is early or increased regenerative braking present themselves. These decreases in energy consumption are indicated in Figure 5(a) and may be made even greater by the unevenness of the alignment.

With regard to the total travel time, as shown in Figure 5(b), there was an initial decrease in travel time as the maximum operating speed was increased, due to an increase in the speed of the cruising regime. However, with the maximum operating speed at around $105 \mathrm{~km} / \mathrm{h}$, the simulator included coasting into the operation to save energy and caused the travel time to increase. Beyond $112 \mathrm{~km} / \mathrm{h}$, the cruising is reduced in favor of regenerative braking so as not to exceed the maximum allowable travel time, resulting in a decrease in travel time. In summary, when the maximum operating speed is low, the train compensates for the lower average speed by operating with minimal coasting to save time. At higher operating speeds, if punctuality is not a concern, coasting is applied to reduce energy consumption, and this results in a reduction of the average speed and an increase in travel time. Since there is a tradeoff between energy saved by coasting and that saved by regenerative braking, there is a critical point while increasing the maximum operating speed where the energy-wise saving through regenerative braking is greater than that saved through coasting. At that point, the simulator reduces the length of the coasting regime, thereby decreasing the travel time and increasing the length of the braking regime and the regenerative braking energy.
6.1.2. Optimal Speed Profiles for Various Allowable Travel Times (Peak). This section demonstrates how the optimal speed profiles vary as the expected travel time varies and how the energy consumption of each train could change as a result of padding the train schedule with extra time. The schedule was altered by adding time in 60 -second increments as well as subtracting time from it in 60-second increments. The resulting optimal speed profiles for each allowable travel time at peak periods are presented in Figure 6.

When the available travel time was significantly reduced below the scheduled time, the train opted for the operation mode according to Scenario III. In this case, the travel time was minimized, and although regenerative braking was included, the resulting energy consumption was substantial. As the available travel time was increased, the train underwent varying levels of coasting along with regenerative braking, thereby reducing the energy consumption. When the available time exceeded the scheduled time, where the speed profile generated was optimal in terms of travel time and energy consumption, as expected, the speed profiles closely resembled the optimal profile. The result is seen in Figure 6 for values of the expected travel time ( $T$ ) equal to $400 \mathrm{~s}, 460 \mathrm{~s}$, and $520 \mathrm{~s}$ almost coinciding.

Table 3 lists the energy consumption of the train with and without the use of the WESS under various values of expected travel times. It also includes the actual travel times with and without the WESS. The results indicate that, with the WESS included, the actual travel times are shorter since the train could include regenerative braking to save energy and this reduces the length of the coasting regime and hence the travel time. However, without the WESS, coasting is the only energy-saving strategy that could be utilized, and therefore, the coasting regime is maximized as much as permitted by the schedule. In addition, since no further energy savings could be obtained from increasing the available travel time, any added time is used to absorb delays caused by service disruption or to facilitate passenger connections to other lines in the network. 


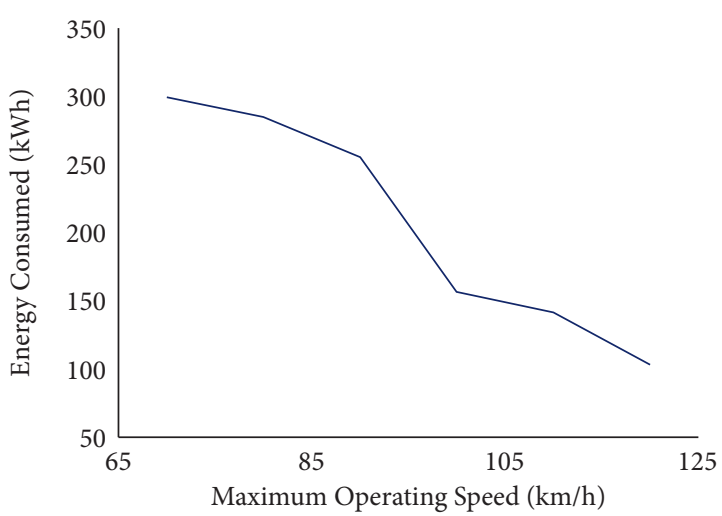

(a)

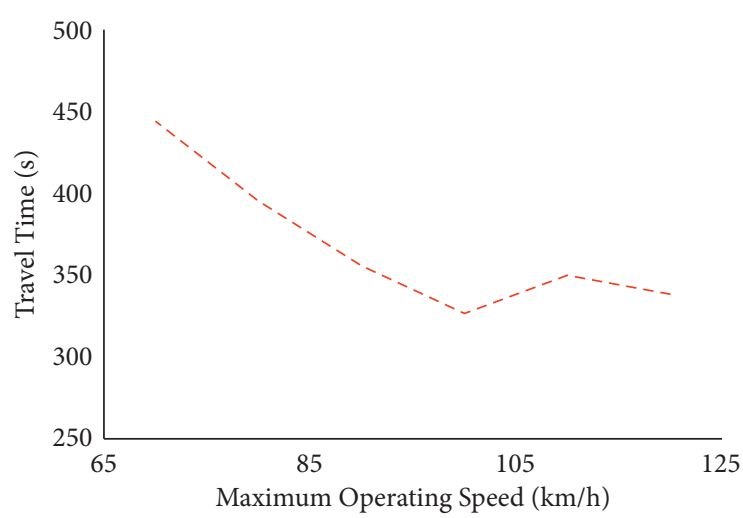

(b)

FIgURE 5: Consumed energy and travel time vs. maximum operating speed. (a) Energy vs. maximum speed. (b) Travel time vs. maximum speed.

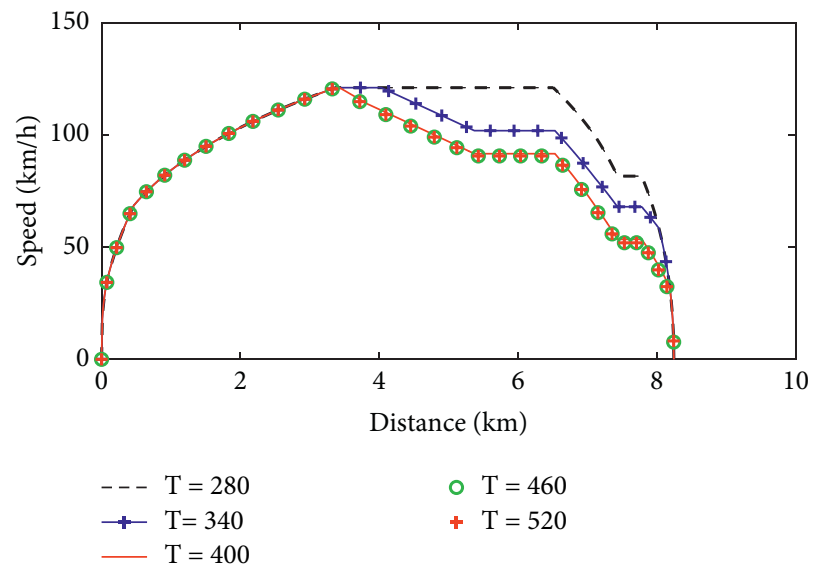

Figure 6: Optimized speed profiles for various expected travel times (seconds).

TABle 3: Minimized energy consumption for various travel times.

\begin{tabular}{|c|c|c|c|c|c|}
\hline \multirow{2}{*}{ Expected travel time (s) } & \multicolumn{2}{|c|}{ Energy consumed (kWh) } & \multirow{2}{*}{ Regenerated energy (kWh) } & \multicolumn{2}{|c|}{ Actual travel time (s) } \\
\hline & With WESS & No WESS & & With WESS & No WESS \\
\hline 280 & 144.1 & 214.4 & 70.3 & 294.8 & 294.8 \\
\hline 340 & 92.8 & 106.1 & 15.8 & 337.2 & 339.3 \\
\hline 400 (schedule) & 84.2 & 83.7 & 7.7 & 346.8 & 375.6 \\
\hline 460 & 84.2 & 83.7 & 3.8 & 373.8 & 426.9 \\
\hline 520 & 84.2 & 83.7 & 3.8 & 374.0 & 427.8 \\
\hline
\end{tabular}

Note. The maximum operating speed $v_{\max }$ is $120 \mathrm{~km} / \mathrm{h}$.

\section{Conclusions}

The purpose of this study was to explore the factors influencing the energy consumption of an electric passenger train and to develop a model which optimized the speed profiles. Simulation analysis was conducted under minimum travel time, coasting only, regenerative braking only, and integration of coasting and regenerative braking scenarios. The speed profiles were optimized, which minimize energy consumption, considering various factors including train schedule and specifications, track alignment, and the availability of WESS. The decision variables were the acceleration at each time step which were varied iteratively by changing the input variables, i.e., maximum operating speed and coasting termination speed. Variations of the inputs resulted in variations of the duration of the motion regimes and, in turn, variations in the energy consumption. The results suggest that if the train was running ahead of schedule, then coasting would be added to the movement and combined synergistically with regenerative braking to optimize energy consumption and minimize operational costs. On the other hand, if the train was late, the coasting regime would be reduced or eliminated to minimize travel time.

In the case study, a train running on a segment of the Long Island Railroad (LIRR) considering peak and off-peak 
passenger volumes and an analysis was conducted to determine the benefits delivered by the WESS. It was found that the optimized speed profile generated by combining coasting and regenerative braking with WESS yielded a $14.3 \%$ and $24.5 \%$ energy saving for off-peak and peak operation, respectively, as against the case where no WESS was included. Since the LIRR operates 760 trains daily, the results indicate that significant savings could be achieved from their electric fleet if the methods outlined in this study are employed. The figures generated could be used for planning purposes to reduce operational costs depending on the schedule and to reduce the carbon footprint of the operator. Another benefit of WESS installation is to provide backup power to take the train to a location where it is safe to evacuate the passengers if there is a loss of power. It can also be used to power continuous station loads such as escalators, lighting, heating/cooling, and charging stations for electric vehicles.

The study may be extended in the future by optimizing the placements of the WESS for a rail line and a network, subject to varying station spacing, track alignment, and posted timetable. On the other hand, the costs of the WESS should be considered in the optimization process to determine the most cost-effective deployment plan.

\section{Notations}

A: $\quad$ Cross-sectional area of the railcar $\left(\mathrm{ft}^{2}\right)$

$a^{t}: \quad$ Applied acceleration at time $t\left(\mathrm{ft} / \mathrm{s}^{2}\right)$

$B^{t}$ : $\quad$ Braking force at time $t$ (lbf)

$b_{c r}:$ Minimum braking distance $(\mathrm{ft})$

$b^{t}: \quad$ Brake rate $(\mathrm{ft} / \mathrm{s})$

$E_{a}$ : $\quad$ Energy consumed during accel. (kWh)

$E_{c}$ : Energy consumed during coasting ( $\left.\mathrm{kWh}\right)$

$E_{r}: \quad$ Total energy regenerated $(\mathrm{kWh})$

$E_{T}: \quad$ Total energy consumed $(\mathrm{kWh})$

$E_{T}^{\prime}$ : $\quad$ Energy consumed in accel. and cruising

$e_{L}: \quad$ Incremental line loss ( $\left.\mathrm{kWh}\right)$

$e_{r}: \quad$ Incremental energy regenerated

$e^{t}: \quad$ Energy consumed at time $t(\mathrm{kWh})$

$F^{t}: \quad$ Tractive effort at time $t(\mathrm{lbf})$

$F_{a}^{t}$ : Adhesive force at time $t(\mathrm{lbf})$

$F_{c}^{t}$ : Tractive force during cruising at time $t$ (lbf)

$F_{m}^{t}:$ Motor force at time $t$ (lbf)

$G^{t}$ : $\quad$ Gradient of alignment section at time $t$ (\%)

$I^{t}: \quad$ Line current at time $t(\mathrm{~A})$

$M_{e}$ : Adjusted mass for rotating components (lbf)

$M: \quad$ Mass of the train (tons)

$N^{t}$ : Normal reaction force (lbf)

$N$ : Number of axles per train unit (axles)

$P_{r}: \quad$ Total motor power $(\mathrm{hp})$

$P^{t}: \quad$ Total power consumption at time $t(\mathrm{kWh})$

$P_{L}^{t}: \quad$ Transmission line losses at time $t$

$R_{D}$ : Remaining distance for safe stop

$R^{t}: \quad$ Train resistance at time $t(\mathrm{lbf})$

$R_{c}^{t}$ : $\quad$ Coasting resistance at time $t(\mathrm{lbf})$

$R_{u}^{t}$ : $\quad$ Unit train resistance at time $t(\mathrm{lbf})$;

$r_{u}: \quad$ Unit line resistance (Ohms)

$r^{t}$ : $\quad$ Line resistance at time $t(\mathrm{Ohms})$
S: $\quad$ Station spacing $(\mathrm{ft})$

$s_{c r}: \quad$ Minimum station spacing $(\mathrm{ft})$

$s^{t}: \quad$ Travel distance in time $t(\mathrm{ft})$

T: $\quad$ Scheduled travel time (s)

$\Delta T: \quad$ Duration of time step (s)

$t_{a}: \quad$ Duration of acceleration regime (s)

$t_{b}$ : Duration of braking regime (s)

$t_{c}$ : Duration of cruising regime (s)

$t_{c o}$ : Duration of coasting regime (s)

$V: \quad$ Line voltage $(\mathrm{V})$

$V_{c}: \quad$ Coasting termination speed $(\mathrm{ft} / \mathrm{s})$

$v^{t}: \quad$ Speed at time $t(\mathrm{ft} / \mathrm{s})$

$v_{\max }$ : Maximum allowable speed $(\mathrm{ft} / \mathrm{s})$

$W: \quad$ Net train weight (tons)

$W: \quad$ Weight per axle (tons)

$\eta: \quad$ Motor efficiency

$\eta_{r}: \quad$ Regeneration coefficient

$\mu^{t}: \quad$ Adhesive coefficient at $t$

$\theta^{t}: \quad$ Alignment inclination at $t$ (degree)

P: Coefficient of rotating masses.

\section{Data Availability}

Some or all the data, models, or code that support the findings in this study are available from the corresponding author upon request. All data applied to this paper has been included in the manuscript. MATLAB code for the algorithm, alignment geometrical profile, train schedules, and passenger demand data and travel patterns are available.

\section{Conflicts of Interest}

The authors declare no conflicts of interest.

\section{References}

[1] J. R. Edwards, Train Energy, Power and Traffic Control, University of Illinois, Urbana-Champaign, IL, USA, 2010.

[2] V. R. Vuchic, Urban Transit: Systems and Technology, John Wiley \& Sons, Hoboken, NJ, USA, 2007.

[3] U.S. Department of Energy Washington, DC 20585 lease include the following information, U.S. Department of Energy Washington, DC 20585, 2020, https://www.connaissancedesenergies. org/sites/default/files/pdf-actualites/may\%202020\%20 energy $\% 2$ Oreport\%20USA.pdf.

[4] U.S. Environmental Protection Agency, "Transportation sector emissions, sources of Greenhouse gas emissions," 2019, https://www.epa.gov/ghgemissions/sources-greenhouse-gasemissions.

[5] U.S. Department of Energy, Transportation Energy Data Book, Oak Ridge National Laboratory, UT-Battelle, Oak Ridge, TN, USA, 2019.

[6] U.S. Environmental Protection Agency, Inventory of U.S. Greenhouse Gas Emissions and Sinks: 1990-2017, https://www. epa.gov/ghgemissions/inventory-us-greenhouse-gasemissions-and-sinks-1990-2017, 2019.

[7] Environmental Information Agency, "US electricity generation by source, amount and share of total in 2017," 2017, https://www.eia.gov/tools/faqs/faq.php?id=427\&t=3.

[8] A. M. Walker, M. U. Lamperth, and S. Wilkins, "On friction braking demand with regenerative braking," Society of 
Automotive Engineers, Hybrid Power Research Group C.A.S.E. Mechanical Engineering Department, Imperial College of Science, Technology and Medicine, London, UK, 2002.

[9] M. Van Gaelen, "Energy efficient Train control in the Netherlands," Analysis of Effects on a Large-Scale Network with Distributed Delays, University of Twente, Utrecht, The Netherlands, 2016.

[10] M. E. Brumbach, Industrial Electricity, Thomson Delmar Learning, New York, NY, USA, 7 edition, 1994.

[11] A. González-Gil, R. Palacin, and P. Batty, "Sustainable urban rail systems: strategies and technologies for optimal management of regenerative braking energy," Energy Conversion and Management, vol. 75, pp. 374-388, 2013.

[12] J. Brandstrom, Optimization for Train Energy Performance, Uppsala University, Uppsala, Sweden, 2014.

[13] K. Kim and S. I.-J. Chien, "Optimal train operation for minimum energy consumption considering track alignment, speed limit, and schedule adherence," Journal of Transportation Engineering, vol. 137, no. 9, pp. 665-674, 2011.

[14] L. Yang, T. Liden, and P. Leander, "Achieving energy efficiency and on-time performance with driver advisory systems," IEEE Conference on Intelligent Rail Transportation, pp. 13-18, 2013.

[15] L. Allen and S. Chien, "Optimization of Rail energy conservation through the adoption of varying coasting strategies: a case study of the Long Island Rail Road's Flatbush branch," in Proceedings of the 93rd Annual Meeting of Transportation Research Board, Washington, DC, USA, January 2014.

[16] H. Liu, M. Zhou, X. Guo, Z. Zhang, and B. Ning, "Timetable optimization for regenerative energy utilization in subway systems," IEEE Transactions on Intelligent Transportation Systems, 2018.

[17] P. Howlett, The Optimal Control of a Train, Centre for Industrial and Applicable Mathematics, University of South Australia, Adelaide, Australia, 2000.

[18] R. Liu and I. M. Golovitcher, "Energy-efficient operation of rail vehicles," Transportation Research Part A: Policy and Practice, vol. 37, no. 10, pp. 917-932, 2003.

[19] S. Su, T. Tang, J. Xun, F. Cao, and Y. Wang, "Design of running grades for energy-efficient train regulation: a case study for Beijing Yizhuang line," IEEE Intelligent Transportation Systems Magazine, vol. 13, no. 2, pp. 189-200, 2021.

[20] Q. Zhu, S. Su, T. Tang, W. Liu, Z. Zhang, and Q. Tian, “An eco-driving algorithm for trains through distributing energy: a Q-Learning approach," ISA Transactions, 2021.

[21] C. Gong, S. Zhang, F. Zhang, J. Jiang, and X. Wang, "An integrated energy-efficient operation methodology for metro systems based on a real case of Shanghai metro line one," Energies, vol. 7, no. 11, pp. 7305-7329, 2014.

[22] S. Su, X. Wang, Y. Cao, and J. Yin, “An energy-efficient train operation approach by integrating the metro timetabling and eco-driving," IEEE Transactions on Intelligent Transportation Systems, vol. 21, 2020.

[23] X. Wang, T. Tang, S. Su, J. Yin, Z. Gao, and N. Lv, “An integrated energy-efficient train operation approach based on the space-time-speed network methodology," Transportation Research Part E, vol. 150, 2021.

[24] P. Liu, L. Yang, Z. Gao, Y. Huang, S. Li, and Y. Gao, "Energyefficient train timetable optimization in the subway system with energy storage devices," IEEE Transactions on Intelligent Transportation Systems, vol. 19, no. 12, pp. 3947-3963, 2018.

[25] X. Luan, Y. Wang, B. De Schutter, L. Meng, G. Lodewijks, and F. Corman, "Integration of real-time traffic management and train control for rail networks-part 2: extensions towards energy-efficient train operations," Transportation Research Part B: Methodological, vol. 115, pp. 72-94, 2018.

[26] Y. Huang, L. Yang, T. Tang, Z. Gao, F. Cao, and K. Li, “Train speed profile optimization with on-board energy storage devices: a dynamic programming based approach," Computers \& Industrial Engineering, vol. 126, pp. 149-164, 2018.

[27] M. Dominguez, A. Fernández-Cardador, A. P. Cucala, and R. R. Pecharroman, "Energy savings in metropolitan railway substations through regenerative energy recovery and optimal design of ATO speed profiles," IEEE Transactions on Automation Science and Engineering, vol. 9, no. 3, pp. 496-504, 2012.

[28] L. A. Allen and S. I. Chien, "Examination of the contributions of coasting and regenerative braking to the efficiency of electric rail vehicles," in Proceedings of the Third International Conference on Railway Technology: Research, Development and Maintenance, Stirlingshire, UK, April 2016.

[29] W. W. Hay, Railroad Engineering, John Wiley \& Sons, New York, NY, USA, 2nd edition, 1982.

[30] S. Sivanagaraju, M. Balasubba Reddy, and D. Srilatha, Generation and Utilization of Electrical Energy, Pearson Education, Delhi, India, 2010. 